\title{
SOLUTION OF STEADY STATE TEMPERATURE PROBLEMS WITH THE AID OF A GENERALIZED FOURIER CONVOLUTION*1
}

\author{
BY \\ A. W. JACOBSON \\ Wayne University
}

1. Introduction. The purpose of this paper is to illistrate the use of the finite Fourier transformation and a generalized convolution in the solution of steady state boundary value problems. Solutions of some basic problems are given in terms of special functions introduced, and by means of a modified Duhamel formula other solutions in turn are expressed as functions of the basic solutions.

The finite sine and cosine transformation are defined by

$$
\begin{aligned}
& S\{F(x)\}=\int_{0}^{\pi} F(x) \sin n x d x=f_{0}(n) \quad(n=1,2, \cdots), \\
& C\{F(x)\}=\int_{0}^{\pi} F(x) \cos n x d x=f_{c}(n) \quad(n=0,1,2, \cdots),
\end{aligned}
$$

respectively. These transformations applied to the derivatives of $F(x)$ yield, for example, the following: $S\left\{F^{\prime}(x)\right\}=-n C\{F(x)\}, S\left\{F^{\prime \prime}(x)\right\}=-n^{2} S\{F(x)\}+n[F(0)-$ $\left.(-1)^{n} F(\pi)\right]$, and $C\left\{F^{\prime}(x)\right\}=n S\{F(x)\}-F(0)+(-1)^{n} F(\pi)$.

If $F(x)$ in $(-2 \pi, 2 \pi)$ and $G(x)$ in $(-\pi, \pi)$, are sectionally continuous functions, then the function

$$
F(x) * G(x)=\int_{-\pi}^{\pi} F(x-y) G(y) d y
$$

is called the convolution of $F$ and $G$ in the interval $(-\pi, \pi)$.

If $F(x)$ is an odd and $G(x)$ is an even sectionally continuous function and if $F(x+2 \pi)=F(x)$, then the product of the transforms can be written in terms of the transform of the convolution, for example, ${ }^{2}$

$$
S\{F(x)\} C\{G(x)\}=\frac{1}{2} S\{F(x) * G(x)\} .
$$

2. A generalized Fourier convolution. Let $F(x, y)$ be a sectionally continuous function of $x$ and $y$ in the square $0 \leq x \leq \pi, 0 \leq y \leq \pi$. A generalized convolution $F^{*}(x)$ of $F(x, y)$ corresponding to the iterated finite sine transformation

$$
\begin{aligned}
S\{S\{F(x, y)\}\} & =\int_{0}^{\pi} \int_{0}^{\pi} F(x, y) \sin n x \sin n^{\prime} y d x d y \\
& =\bar{f}\left(n, n^{\prime}\right) \quad\left(n, n^{\prime}=1,2, \cdots\right)
\end{aligned}
$$

is defined by

$$
F^{*}(x)=-\int_{-\pi}^{\pi} F_{1}(x-y, y) d y
$$

*Received August 24, 1948.

1Presented to the American Mathematical Society April 15, 1948. Also see abstract in Bull. Am. Math. Society, vol. 54. The author expresses his thanks to Professor R. V. Churchill for many valuable suggestions.

${ }^{2}$ R. V. Churchill, Modern Operational Mathematics in Engineering, McGraw-Hill, 1944, p. 274-276. 
where $F_{1}(x, y)$ is an odd periodic extension of $F$ with respect to $x$ and an odd extension with respect to $y$.

When $n^{\prime}=n$, it can be shown ${ }^{3}$ that

$$
S\{S\{F(x, y)\}\}=\frac{1}{2} C\left\{F^{*}(x)\right\} .
$$

In case $F(x, y)=F_{1}(x) G_{1}(y)$, the function $F^{*}(x)$ is the convolution (1) of $F_{1}$ and $G_{1}$.

3. Special functions. In this section two key functions are introduced as well as their sine and cosine transforms. In the sequel solutions of some basic boundary value problems are expressed in terms of these functions.

If $|r|<1$, then $\log \left(1+\mathrm{re}^{i \theta}\right)=-\sum_{\nu=1}^{\infty}(-r)^{\nu} e^{i \nu \theta} / \nu$. Also if $R^{2}=(1+r \cos \theta)^{2}+$ $r^{2} \sin ^{2} \theta$ and $\tan \Phi=r \sin \theta /(1+r \cos \theta)$, then

$$
\log \left(1+\mathrm{re}^{i \theta}\right)=\log \operatorname{Re}^{i \Phi}=\log R+i \Phi
$$

Equating the imaginary parts of (5), we get

$$
\arctan \frac{r \sin \theta}{1+r \cos \theta}=-\sum_{r=1}^{\infty} \frac{(-r)^{\prime} \sin \nu \theta}{\nu} .
$$

When $|r| \leq 1$, the series is a Fourier sine series. The sine transforms of the function are, except for the normalizing factor $\pi / 2$, the Fourier sine coefficients, i.e.,

$$
S\left\{\arctan \frac{r \sin x}{1+r \cos x}\right\}=-\frac{\pi}{2} \frac{(-1)^{n} r^{n}}{n}, \quad|r| \leq 1,
$$

where $\theta$ has been replaced by $x$. Since $(-1)^{n+1} f_{\bullet}(n)=S\{F(\pi-x)\}$, then

$$
S\left\{\arctan \frac{r \sin x}{1-r \cos x}\right\}=\frac{\pi}{2} \frac{r^{n}}{n} \text {. }
$$

Setting $r=e^{-z}$, the above formula becomes

$$
S\left\{\arctan \frac{\sin x}{e^{z}-\cos x}\right\}=\frac{\pi}{2} \frac{e^{-n z}}{n}, \quad z \geq 0 .
$$

Let $b_{0}(n, u)$ denote the function

$$
\frac{\sinh n u}{n \sinh n \pi}=\sum_{\nu=0}^{\infty} \frac{1}{n}\{\exp \{-[(2 \nu+1) \pi-u] n\}-\exp \{-[(2 \nu+1) \pi+u] n\}\} .
$$

Let the inverse sine transform of the function $b_{0}(n, u), S^{-1}\left\{b_{0}(n, u)\right\}$, be $B_{01}(x, u)$, then according to formula (6), when $\pi-u \geq 0$,

$$
\begin{aligned}
B_{01}(x, u)=\sum_{\nu=0}^{\infty} & {\left[\arctan \frac{\sin x}{\exp \{(2 \nu+1) \pi-u\}-\cos x}\right.} \\
& \left.-\arctan \frac{\sin x}{\exp \{(2 \nu+1) \pi+u\}-\cos x}\right] \\
= & \sum_{\nu=0}^{\infty} \arctan \frac{\sin x \sinh u}{\cosh (2 \nu+1) \pi-\cos x \cosh u} .
\end{aligned}
$$

${ }^{8}$ A. W. Jacobson, A Generalized Convolution for the Finite Fourier Transformations, Thesis, University of Michigan, 1948, p. 9-12. In the sequel reference to this thesis will be marked [3]. 
Equating the real parts of (5), we have

$$
\log \frac{1}{1+2 r \cos x+r^{2}}=2 \sum_{\nu=1}^{\infty} \frac{(-1)^{\nu} r^{\nu}}{\nu} \cos \nu x .
$$

Hence the cosine transformation yields

$$
\begin{aligned}
C\left\{\log \frac{1}{1+2 r \cos x+r^{2}}\right\} & =\frac{\pi(-1)^{n} r^{n}}{n}, & & n=1,2, \cdots \\
& =0 & & n=0
\end{aligned}
$$

Since $(-1)^{n} f_{c}(n)=C\{F(\pi-x)\}$, and setting $r=e^{-z}$, we get when $z \geq 0$

$$
\begin{array}{rlrl}
C\left\{\log \frac{e^{z}}{2[\cosh z-\cos x]}\right\} & =\frac{\pi e^{-n z}}{n}, & & n=1,2, \cdots \\
& =0 & n=0 .
\end{array}
$$

Let $b_{1}(n, u)$ denote the function

$$
\frac{\cosh n u}{n \sinh n \pi}=\sum_{\nu=0}^{\infty} \frac{1}{n}\{\exp \{-[(2 \nu+1) \pi-u] n\}+\exp \{-[(2 \nu+1) \pi+u] n\}\} .
$$

And let $B_{12}(x, u)$ denote the inverse cosine transform $C^{-1}\left\{b_{1}(n, u)\right\}$ of the function $b_{1}(n, u)$. When $\pi-u \geq 0$, according to formula (9), we get

$$
B_{12}(x, u)=\frac{1}{\pi} \log \prod_{\nu=0}^{\infty} \frac{\exp \{2(2 \nu+1) \pi\}}{4[\cosh (2 \nu \pi+\pi-u)-\cos x][\cosh (2 \nu \pi+\pi+u)-\cos x]} .
$$

4. Problems in two dimensions. Basic problems (A). Let $U_{0}(x, y)$ be the solution of the following steady state temperature problem in the region $R: 0 \leq x \leq \pi, 0 \leq y \leq \pi$ :

$$
\begin{aligned}
& \frac{\partial^{2} U_{0}}{\partial x^{2}}+\frac{\partial^{2} U_{0}}{\partial y^{2}}=0 \quad \text { in } \quad R, \\
& U_{0}(+0, y)=U_{0}(\pi-0, y)=0, \quad 0<y<\pi, \\
& U_{0}(x,+0)=\frac{\pi-x}{\pi}, \quad U_{0}(x, \pi-0)=0, \quad 0<x<\pi .
\end{aligned}
$$

The sine transformation of problem (A) with respect to $x$ yields

$$
\begin{aligned}
& \frac{d^{2} u_{0}}{d y^{2}}-n^{2} u_{0}(n, y)=0, \\
& u_{0}(n, 0)=\frac{1}{n}, \quad u_{0}(n, \pi)=0 .
\end{aligned}
$$

The solution of the transformed problem is

$$
u_{0}(n, y)=\frac{\sinh n(\pi-y)}{n \sinh n \pi} .
$$


In the previous section, formula (7), this function was designated by the function $b_{0}(n, u)$, i.e.,

$$
u_{0}(n, y)=b_{0}(n, \pi-y) .
$$

According to formula (8), its inverse sine transform is the function $B_{01}(x, \pi-y)$, so that

$$
U_{0}(x, y)=B_{01}(x, \pi-y)
$$

Problem (B)

$$
\begin{array}{ll}
\frac{\partial^{2} U}{\partial x^{2}}+\frac{\partial^{2} U}{\partial y^{2}}=0 \quad \text { in } \quad R & \\
U(+0, y)=U(\pi-0, y)=0, & 0<y<\pi, \\
U(x,+0)=H(x), \quad U(x, \pi-0)=0, & 0<x<\pi .
\end{array}
$$

The transformed problem is

$$
\begin{aligned}
& \frac{d^{2} u}{d y^{2}}-n^{2} u(n, y)=0, \\
& u(n, 0)=h(n), \quad u(n, \pi)=0 .
\end{aligned}
$$

Upon multiplying the equations in problem $\left(\mathrm{A}^{\prime}\right)$ by the function $n h(n)$ of the parameter, it is evident that the product $n h(n) u_{0}(n, y)$ is also a solution of problem $\left(\mathrm{B}^{\prime}\right)$. If the solution of the latter is unique then

$$
u(n, y)=n h(n) u_{0}(n, y)
$$

Since $U_{0}(0, y)=U_{0}(\pi, y)=0$, then $n S\{U(x, y)\}=C\left\{\partial / \partial x U_{0}(x, y)\right\}$. Hence

$$
S\{U(x, y)\}=S\{H(x)\} n S\left\{U_{0}(x, y)\right\} .
$$

becomes

$$
S\{U(x, y)\}=S\{H(x)\} C\left\{\frac{\partial}{\partial x} U_{0}(x, y)\right\} .
$$

The product of the transforms here can be written, according to formula (2), as the sine transform of the convolution of the two functions, and making the inverse transformation, there results

$$
U(x, y)=\frac{1}{2} H(x)^{*} \frac{\partial}{\partial x} U_{0}(x, y) .
$$

Since $U_{0}(x, y)=B_{01}(x, \pi-y)$, equation (12), the last result becomes in terms of the convolution integral (1)

$$
U(x, y)=\frac{1}{2} \int_{-\pi}^{\pi} H(x-\lambda) \frac{\partial}{\partial \lambda} B_{01}(\lambda, \pi-y) d \lambda .
$$

In view of formula (11) $\partial / \partial \lambda B_{01}(\lambda, \pi-y)$ is an even function of $\lambda$, hence $H$ is to be extended to $(-\pi, 0)$ as an odd function. 
Problem (C).

$$
\begin{array}{ll}
\frac{\partial^{2} U}{\partial x^{2}}+\frac{\partial^{2} U}{\partial y^{2}}=F(x, y) \quad \text { in } & R \\
U(+0, y)=U(\pi-0, y)=0, & 0<y<\pi \\
U(x,+0)=U(x, \pi-0)=0, & 0<x<\pi .
\end{array}
$$

The sine transformation of this problem with respect to $x$ yields

$$
\begin{aligned}
& \frac{d^{2} u}{d y^{2}}-n^{2} u(n, y)=f(n, y) \\
& u(n, 0)=u(n, \pi)=0 .
\end{aligned}
$$

The solution of the transformed problem, in terms of the Green's function $g(n, y, \mu)$, is

$$
u(n, y)=\int_{0}^{\pi} g(n, y, \mu) f(n, \mu) d \mu
$$

where

$$
\begin{array}{rlrl}
g(n, y, \mu)=\frac{\sinh n \mu \sinh n(\pi-y)}{n \sinh n \pi}, & & \mu \leq y \\
& =\frac{\sinh n y \sinh n(\pi-\mu)}{n \sinh n \pi}, & & \mu \geq y,
\end{array}
$$

or

$$
\begin{aligned}
g(n, y, \mu) & =\frac{\cosh n(\pi-y-\mu)}{2 n \sinh n \pi}-\frac{\cosh n(\pi-y+\mu)}{2 n \sinh n \pi}, & & \mu \leq y \\
& =\frac{\cosh n(\pi-y-\mu)}{2 n \sinh n \pi}-\frac{\cosh n(\pi-\mu+y)}{2 n \sinh n \pi}, & & \mu \geq y .
\end{aligned}
$$

According to formula (10) this can be written

$$
\begin{array}{rlrl}
g(n, y, \mu) & =\frac{1}{2} b_{1}(n, \pi-y-\mu)-\frac{1}{2} b_{1}(n, \pi-y+\mu), & & \mu \leq y \\
& =\frac{1}{2} b_{1}(n, \pi-y-\mu)-\frac{1}{2} b_{1}(n, \pi-\mu+y), & \mu \geq y .
\end{array}
$$

The inverse cosine transform of $b_{1}(n, u)$ is, in view of formula (11), the function $B_{12}(x, u)$. Hence

$$
G(x, y, \mu)=\frac{1}{2} B_{12}(x, \pi-y-\mu)-\frac{1}{2} B_{12}(x, \pi-y+\mu) .
$$

where

$$
C^{-1}\{g(n, y, \mu)\}=G(x, y, \mu) .
$$

Writing solution (13) as

$$
S\{U(x, y)\}=\int_{0}^{\pi} C\{G(x, y, \mu)\} S\{F(x, \mu)\} d \mu,
$$


and replacing the product of the transforms by the sine transform of the convolution and making the inverse transformation, we obtain

$$
U(x, y)=\frac{1}{2} \int_{0}^{\pi} G(x, y, \mu)^{*} F(x, \mu) d \mu,
$$

or

$$
U(x, y)=\frac{1}{2} \int_{0}^{\pi} \int_{-\pi}^{\pi} G(x-\lambda, y, \mu) F(\lambda, \mu) d \lambda d \mu .
$$

Substituting for $G$ from (14), there results

$$
U(x, y)=\frac{1}{4} \int_{0}^{\pi} \int_{-\pi}^{\pi}\left[B_{12}(x-\lambda, \pi-y-\mu)-B_{12}(x-\lambda, \pi-y+\mu)\right] F(\lambda, \mu) d \lambda d \mu .
$$

The function $B_{12}(x, u)$ is an even function of $x$, hence $H(x, y)$ must be extended to $(-\pi, 0)$ as an odd function of $x$.

The above solutions can be shown to satisfy the respective boundary value problems for a wide class of functions involved. See [3] p. 58-75.

5. Resolution of boundary value problems with the aid of a generalized Fourier convolution. Let $U(x, y)$ be the steady state temperature in the region $R: 0 \leq x \leq \pi$, $0 \leq y \leq \pi, 0 \leq z \leq \pi$, satisfying the following conditions:

$$
\begin{aligned}
& \frac{\partial^{2} U}{\partial x^{2}}+\frac{\partial^{2} U}{\partial y^{2}}+\frac{\partial^{2} U}{\partial z^{2}}=F(x, y, z) \quad \text { in } \quad R, \\
& U(0, y, z)=H_{1}(y, z), \quad U(\pi, y, z)=0, \\
& U(x, 0, z)=H_{3}(x, y), \quad U(x, \pi, z)=0, \\
& U(x, y, 0)=H_{5}(x, y), \quad U(x, y, \pi)=0 .
\end{aligned}
$$

Three of the boundary conditions have been written here as homogeneous since this involves no loss in generality. The sine transform of this problem with respect to $x$ is

$$
\begin{aligned}
& -n^{2} u(n, y, z)+n H_{1}(y, z)+\frac{\partial^{2} u}{\partial y^{2}}+\frac{\partial^{2} u}{\partial z^{2}}=f(n, y, z), \\
& u(n, 0, z)=h_{3}(n, z), \quad u(n, \pi, z)=0, \\
& u(n, y, 0)=h_{5}(n, y), \quad u(n, y, \pi)=0 .
\end{aligned}
$$

Nowlet $V\left(x, x^{\prime}, y, z\right)$, depending on the parameter $x^{\prime}$ independent of $x, y, z$, be the solution of the following auxiliary problem:

$$
\begin{array}{ll}
\frac{\partial^{2} V}{\partial x^{2}}+\frac{\partial^{2} V}{\partial y^{2}}+\frac{\partial^{2} V}{\partial z^{2}}=\frac{\pi-x}{\pi} F\left(x^{\prime}, y, z\right) \quad \text { in } \quad R, \\
V\left(0, x^{\prime}, y, z\right)=\frac{\pi-x^{\prime}}{\pi} H_{1}(y, z), & V\left(\pi, x^{\prime}, y, z\right)=0, \\
V\left(x, x^{\prime}, 0, z\right)=\frac{\pi-x}{\pi} H_{3}\left(x^{\prime}, z\right), & V\left(x, x^{\prime}, \pi, z\right)=0, \\
V\left(x, x^{\prime}, y, 0\right)=\frac{\pi-x}{\pi} H_{5}\left(x^{\prime}, y\right), & V\left(x, x^{\prime}, y, \pi\right)=0 .
\end{array}
$$


The sine transform of $(\mathrm{E})$ with respect to $x$ is

$$
\begin{aligned}
& -n^{2} v\left(n, x^{\prime}, y, z\right)+n \frac{\pi-x^{\prime}}{\pi} H_{1}(y, z)+\frac{\partial^{2} v}{\partial y^{2}}+\frac{\partial^{2} v}{\partial z^{2}}=\frac{1}{n} F\left(x^{\prime}, y, z\right), \\
& v\left(n, x^{\prime}, 0, z\right)=\frac{1}{n} H_{3}\left(x^{\prime}, z\right), \quad v\left(n, x^{\prime}, \pi, z\right)=0, \\
& v\left(n, x^{\prime}, y, 0\right)=\frac{1}{n} H_{5}\left(x^{\prime}, y\right), \quad v\left(n, x^{\prime}, y, \pi\right)=0 .
\end{aligned}
$$

Next let $\bar{v}\left(n, n^{\prime}, z\right)$ be the sine transform of $v\left(n, x^{\prime}, y, z\right)$ with respect to $x^{\prime}$. When $n^{\prime}=n$, we get

$$
\begin{aligned}
& -n^{2} \bar{v}(n, y, z)+H_{1}(y, z)+\frac{\partial^{2} \bar{v}}{\partial y^{2}}+\frac{\partial^{2} \bar{v}}{\partial z^{2}}=\frac{1}{n} f(n, y, z), \\
& \bar{v}(n, 0, z)=\frac{1}{n} h_{3}(n, z), \quad \bar{v}(n, \pi, z)=0, \\
& \bar{v}(n, y, 0)=\frac{1}{n} h_{5}(n, y), \quad \bar{v}(n, y, \pi)=0 .
\end{aligned}
$$

If we now multiply the equations in $\left(\mathrm{E}^{\prime}\right)$ by the parameter $n$, we note that problems $\left(\mathrm{D}^{\prime}\right)$ and $\left(\mathrm{E}^{\prime}\right)$ are equivalent, and

$$
u(n, y, z)=n \bar{v}(n, y, z)
$$

Since $\bar{v}(n, y, z)$ is the iterated sine transform of $V\left(x, x^{\prime}, y, z\right)$, it follows, according formula (4), that

$$
\begin{aligned}
u(n, y, z) & =n C\left\{\frac{1}{2} V^{*}(x, y, z)\right\} . \\
& =-S\left\{\frac{1}{2} \frac{\partial}{\partial x} V^{*}(x, y, z)\right\}
\end{aligned}
$$

And therefore

$$
U(x, y, z)=-\frac{1}{2} \frac{\partial}{\partial x} V^{*}(x, y, z),
$$

which can be written (formula 3)

$$
U(x, y, z)=\frac{1}{2} \frac{\partial}{\partial x} \int_{-\pi}^{\pi} V\left(x-x^{\prime}, x^{\prime}, y, z\right) d x^{\prime} .
$$

Formula (16) is the Duhamel integral extended from time to space coordinates.

Problem (D) can further be resolved into simpler problems. We first note that problem (E) can be written as

$$
V\left(x, x^{\prime}, y, z\right)=\frac{\pi-x}{\pi} V_{1}\left(x^{\prime}, y, z\right)+V_{2}\left(x, x^{\prime}, y, z\right)+V_{3}\left(x, x^{\prime}, y, z\right),
$$


where $V_{1}, V_{2}$ and $V_{3}$ are solutions of the problems:

$$
\begin{aligned}
& \frac{\partial^{2} V_{1}}{\partial y^{2}}+\frac{\partial^{2} V_{1}}{\partial z^{2}}=0, \\
& V_{1}\left(x^{\prime}, 0, z\right)=H_{3}\left(x^{\prime}, z\right), \quad V_{1}\left(x^{\prime}, \pi, z\right)=0, \\
& V_{1}\left(x^{\prime}, y, 0\right)=H_{5}\left(x^{\prime}, y\right), \quad V_{1}\left(x^{\prime}, y, \pi\right)=0 ; \\
& \frac{\partial^{2} V_{2}}{\partial x^{2}}+\frac{\partial^{2} V_{2}}{\partial y^{2}}+\frac{\partial^{2} V_{2}}{\partial z^{2}}=0, \\
& V_{2}\left(0, x^{\prime}, y, z\right)=\frac{\pi-x^{\prime}}{\pi} H_{1}(y, z)-V_{1}\left(x^{\prime}, y, z\right) \\
& V_{2}\left(\pi, x^{\prime}, y, z\right)=0, \\
& V_{2}\left(x, x^{\prime}, 0, z\right)=V_{2}\left(x, x^{\prime}, \pi, z\right)=0, \\
& V_{2}\left(x, x^{\prime}, y, 0\right)=V_{2}\left(x, x^{\prime}, y, \pi\right)=0 ;
\end{aligned}
$$

and

$$
\begin{aligned}
& \frac{\partial^{2} V_{3}}{\partial x^{2}}+\frac{\partial^{2} V_{3}}{\partial y^{2}}+\frac{\partial^{2} V_{3}}{\partial z^{2}}=\frac{\pi-x}{\pi} F\left(x^{\prime}, y, z\right), \\
& V_{3}\left(0, x^{\prime}, y, z\right)=V_{3}\left(\pi, x^{\prime}, y, z\right)=0, \\
& V_{3}\left(x, x^{\prime}, 0, z\right)=V_{3}\left(x, x^{\prime}, \pi, z\right)=0, \\
& V_{3}\left(x, x^{\prime}, y, 0\right)=V_{3}\left(x, x^{\prime}, y, \pi\right)=0 .
\end{aligned}
$$

It can be'readily shown, see [3] p. 19-23, that the solution $U(x, y, z)$ of problem (D) then takes the following form:

$$
U(x, y, z)=V_{1}(x, y, z)-\frac{1}{2} \frac{\partial}{\partial x} V_{2}^{*}(x, y, z)-\frac{1}{2} \frac{\partial}{\partial x} V_{3}^{*}(x, y, z) .
$$

The solution $V_{1}$ of the two dimensional problem (a) is the sum of solutions of the type of problem (B), Sec. 4, that is,

$$
\begin{aligned}
V_{1}\left(x^{\prime}, y, z\right)=\frac{1}{2} & H_{3}\left(x^{\prime}, z\right) * \frac{\partial}{\partial z} B_{01}(y, \pi-z) \\
& +\frac{1}{2} H_{5}\left(x^{\prime}, y\right) * \frac{\partial}{\partial y} B_{01}(y, \pi-z)
\end{aligned}
$$

We shall next proceed to obtain the solution $V_{2}$ of problem (b). Let $v_{2}\left(x, x^{\prime}, m, z\right)$ be the sine transform of $V_{2}\left(x, x^{\prime}, y, z\right)$ with respect to $y$ and also let $\bar{v}_{2}\left(x, x^{\prime}, m, p\right)$ be 
the iterated transform of $V_{2}\left(x, x^{\prime}, y, z\right)$ with respect to $y$ and $z$. Then applying this iterated transformation to problem (b) we get

$$
\begin{gathered}
\frac{\partial^{2} \bar{v}_{2}}{\partial x^{2}}-\left(m^{2}+p^{2}\right) \bar{v}_{2}\left(x, x^{\prime}, m, p\right)=0, \\
\bar{v}_{2}\left(0, x^{\prime}, m, p\right)=\bar{q}\left(x^{\prime}, m, p\right), \quad \bar{v}_{2}\left(\pi, x^{\prime}, m, p\right)=0,
\end{gathered}
$$

where $\bar{q}\left(x^{\prime}, m, p\right)=S\left\{q\left(x^{\prime}, m, z\right)\right\}$.

We shall now formulate the following basic problem (d) in three dimensions, and then express the solution $V_{2}$ in terms of its solution:

$$
\begin{aligned}
& \frac{\partial^{2} U_{0}}{\partial x^{2}}+\frac{\partial^{2} U_{0}}{\partial y^{2}}+\frac{\partial^{2} U_{0}}{\partial z^{2}}=0, \\
& U_{0}(0, y, z)=\frac{\pi-y}{\pi} \cdot \frac{\pi-z}{\pi}, \quad U_{0}(\pi, y, z)=0, \\
& U_{0}(x, 0, z)=U_{0}(x, \pi, z)=0, \\
& U_{0}(x, y, 0)=U_{0}(x, y, \pi)=0 .
\end{aligned}
$$

Let $u_{0}(x, m, z)$ and $\bar{u}_{0}(x, m, p)$, respectively, be the sine transform of $U_{0}(x, y, z)$ with respect to $y$ and its iterated transform with respect to $y$ and $z$. Then

$$
\begin{aligned}
& \frac{\partial^{2} \bar{u}_{0}}{\partial x^{2}}-\left(m^{2}+p^{2}\right) \bar{u}_{0}(x, m, p)=0, \\
& \bar{u}_{0}(0, m, p)=\frac{1}{m p}, \quad \bar{u}_{0}(\pi, m, p)=0 .
\end{aligned}
$$

If we multiply the equations in problem $\left(\mathrm{d}^{\prime}\right)$ by the function of the parameters $m p \bar{q}\left(x^{\prime}, m, p\right)$, it is evident in view of problem $\left(\mathrm{b}^{\prime}\right)$ that

That is

$$
\bar{v}_{2}\left(x, x^{\prime}, m, p\right)=\bar{q}\left(x^{\prime}, m, p\right) m p \bar{u}_{0}(x, m, p) .
$$

$$
S\left\{v_{2}\left(x, x^{\prime}, m, z\right)\right\}=S\left\{q\left(x^{\prime}, m, z\right)\right\} m p S\left\{u_{0}(x, m, z)\right\} .
$$

Since $u_{0}(x, m, 0)=u_{0}(x, m, \pi)=0$, then $p S\left\{u_{0}(x, m, z)\right\}=C\left\{\partial u_{0} / \partial z(x, m, z)\right\}$. Hence the product of the transforms can be replaced by the sine transform of the convolution of the two functions. Upon applying the inverse transformation, we get

$$
\begin{aligned}
v_{2}\left(x, x^{\prime}, m, z\right) & =\frac{1}{2} q\left(x^{\prime}, m, z\right)^{*} m \frac{\partial}{\partial z} u_{0}(x, m, z) \\
& =\frac{1}{2} \int_{-\pi}^{\pi} q\left(x^{\prime}, m, z-\lambda\right) m \frac{\partial}{\partial \lambda} u_{0}(x, m, \lambda) d \lambda,
\end{aligned}
$$

which in turn can be written formally as follows:

$$
S\left\{V_{2}\left(x, x^{\prime}, y, z\right)\right\}=\frac{1}{2} \int_{-\pi}^{\pi} S\{Q(x, y, z-\lambda)\} m S\left\{\frac{\partial}{\partial \lambda} U_{0}(x, y, \lambda)\right\} d \lambda .
$$


Since again $\partial / \partial \lambda U_{0}(x, 0, \lambda)=\partial / \partial \lambda U_{0}(x, \pi, \lambda)=0$, then, repeating the steps above, we obtain

$$
V_{2}\left(x, x^{\prime}, y, z\right)=\frac{1}{4} \int_{-\pi}^{\pi} \int_{-\pi}^{\pi} Q(x, y-\mu, z-\lambda) \frac{\partial^{2}}{\partial \lambda \partial \mu} U_{0}(x, \mu, \lambda) d \lambda d \mu .
$$

The solution $U_{0}(x, y, z)$ can be obtained with the aid of the Fourier sine series. The solution of problem $\left(d^{\prime}\right)$ is

$$
\bar{u}_{0}(x, m, p)=\frac{\sinh (\pi-x)\left(m^{2}+p^{2}\right)^{1 / 2}}{m p \sinh \pi\left(m^{2}+p^{2}\right)^{1 / 2}}
$$

These functions are, except for the constant factor $\pi / 2$, the Fourier sine coefficients for the function $u_{0}(x, m, z)$, which in turn are in like manner the sine coefficients for the function $U_{0}(x, y, z)$. Hence

$$
U_{0}(x, y, z)=\frac{4}{\pi^{2}} \sum_{m=1}^{\infty} \sum_{p=1}^{\infty} \frac{\sinh (\pi-x)\left(m^{2}+p^{2}\right)^{1 / 2}}{m p \sinh \pi\left(m^{2}+p^{2}\right)^{1 / 2}} \sin p z \sin m y .
$$

Solution of problem (c). The iterated sine transform of (c) with respect to $x$ and $y$ is

$$
\begin{aligned}
& \frac{\partial^{2} \bar{v}_{3}}{\partial x^{2}}-\left(n^{2}+m^{2}\right) \bar{v}_{3}\left(n, x^{\prime}, m, z\right)=\frac{1}{n} f\left(x^{\prime}, m, z\right), \\
& \bar{v}_{3}\left(n, x^{\prime}, m, 0\right)=\bar{v}_{3}\left(n, x^{\prime}, m, \pi\right)=0 .
\end{aligned}
$$

In terms of the Green's function $g(n, m, z, \mu)$ the solution of $\left(\mathrm{c}^{\prime}\right)$ has the form

$$
\bar{v}_{3}\left(n, x^{\prime}, m, z\right)=\int_{0}^{\pi} \frac{g(n, m, z, \mu) f\left(x^{\prime}, m, \mu\right) d \mu}{n},
$$

where

$$
g(n, m, z, \mu)=\frac{\sinh \mu\left(m^{2}+n^{2}\right)^{1 / 2} \sinh (z-\pi)\left(m^{2}+n^{2}\right)^{1 / 2}}{n m \sinh \pi\left(m^{2}+n^{2}\right)^{1 / 2}}, \quad \mu \leq z,
$$

and with $\mu$ and $z$ interchanged when $\mu \geq z$.

Performing the iterated inverse sine transformation with the aid of Fourier sine series, we get

$$
V_{3}\left(x, x^{\prime}, y, z\right)=\frac{4}{\pi^{2}} \sum_{n=1}^{\infty} \sum_{m=1}^{\infty} \bar{v}_{3}\left(n, x^{\prime}, m, z\right) \sin n x \sin m y,
$$

where $\bar{v}_{3}\left(n, x^{\prime}, m, z\right)$ is defined by $(17)$. 